This item was submitted to Loughborough's Research Repository by the author.

Items in Figshare are protected by copyright, with all rights reserved, unless otherwise indicated.

\title{
A new extension of fuzzy sets using rough sets: R-fuzzy sets
}

PLEASE CITE THE PUBLISHED VERSION

http://dx.doi.org/10.1016/j.ins.2009.10.004

PUBLISHER

(c) Elsevier

VERSION

AM (Accepted Manuscript)

LICENCE

CC BY-NC-ND 4.0

REPOSITORY RECORD

Hinde, Chris J., and Yingjie Yang. 2019. "A New Extension of Fuzzy Sets Using Rough Sets: R-fuzzy Sets". figshare. https://hdl.handle.net/2134/13244. 
This item was submitted to Loughborough's Institutional Repository (https://dspace.lboro.ac.uk/) by the author and is made available under the following Creative Commons Licence conditions.

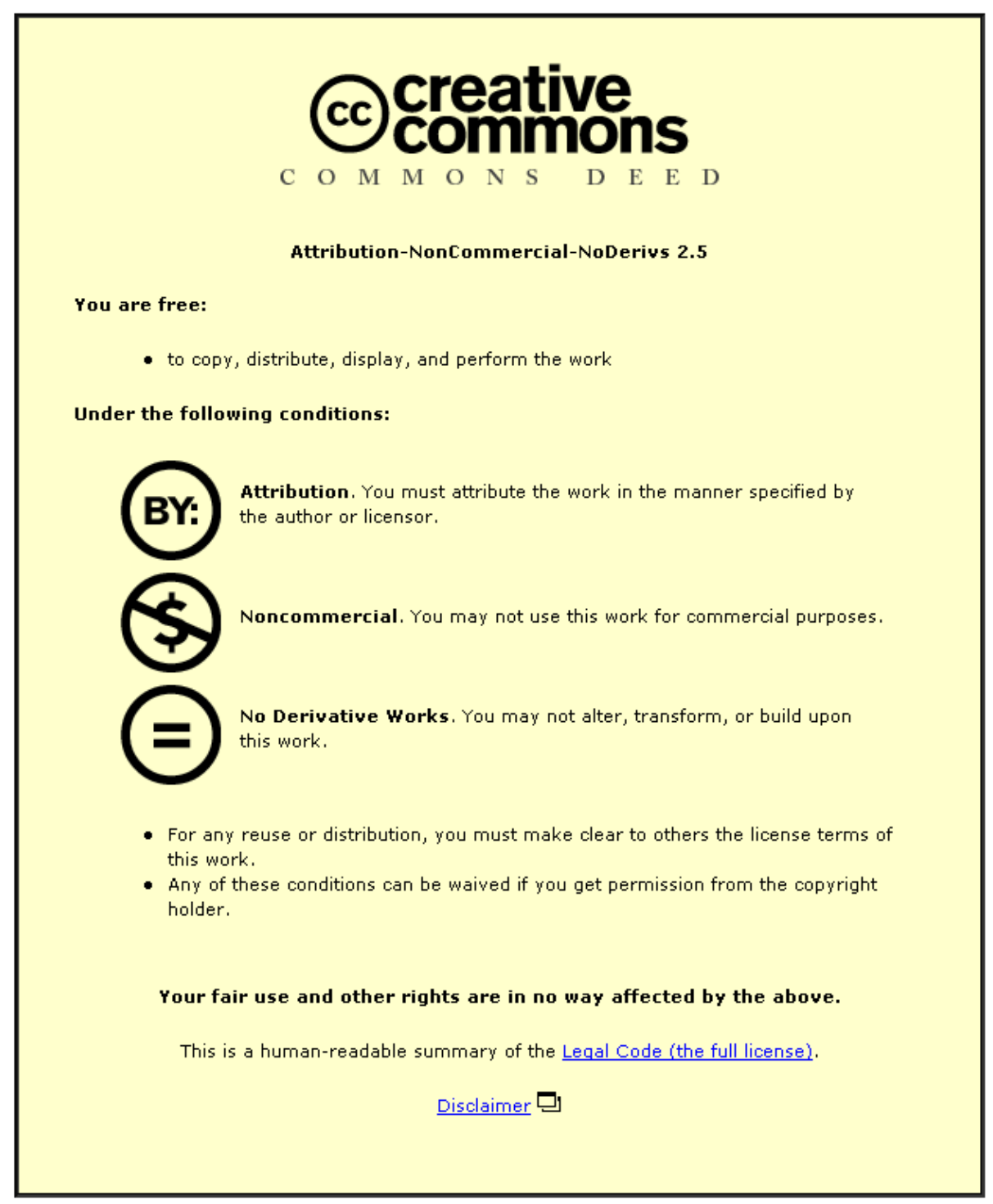

For the full text of this licence, please go to: http://creativecommons.org/licenses/by-nc-nd/2.5/ 


\title{
A new extension of fuzzy sets using rough sets: R-Fuzzy Sets
}

\author{
Yingjie Yang ${ }^{\mathrm{a}, *}$, Chris Hinde ${ }^{\mathrm{b}}$ \\ ${ }^{a}$ Centre for Computational Intelligence, De Montfort University, The Gateway, \\ Leicester, LE1 9BH, UK \\ ${ }^{\mathrm{b}}$ Department of Computer Science,Loughborough University, \\ Loughborough, LE11 3TU, UK
}

\begin{abstract}
This paper presents a new extension of fuzzy sets: R-fuzzy sets. The membership of an element of a R-fuzzy set is represented as a rough set. This new extension facilitates the representation of an uncertain fuzzy membership with a rough approximation. Based on our definition of R-fuzzy sets and their operations, the relationships between R-fuzzy sets and other fuzzy sets are discussed and some examples are provided.
\end{abstract}

Key words: Fuzzy Sets, Rough sets, R-fuzzy sets

* Corresponding author.

Email addresses: yyang@dmu.ac.uk (Yingjie Yang), C.J.Hinde@lboro.co.uk (Chris Hinde). 


\section{Introduction}

Various uncertainties in real world applications can bring difficulties in determining the crisp membership functions of type-1 fuzzy sets [25]. They involve not only vagueness (lack of sharp class boundaries), but also ambiguity (lack of information). Hence many extensions have been developed to represent these uncertainties in membership values, such as interval-valued fuzzy sets [35], Atanassov intuitionistic fuzzy sets [1], shadowed sets [32] and type-2 fuzzy sets [25]. These models introduce intervals, multiple parameters, and fuzzy sets to describe the uncertain membership functions of fuzzy sets. However, intervals and multiple parameters in interval-valued fuzzy sets, Atanassov intuitionistic fuzzy sets and shadowed sets do not recognise the difference between values within their intervals or shadowed zone. A type-2 fuzzy set describes its memberships using type-1 fuzzy sets, but it needs precise crisp values to describe its secondary memberships. Here, we adopt rough sets to approximate the uncertain fuzzy membership function of a fuzzy set. Rough sets provide a different model in representing ambiguity of sets, and the combination of fuzzy sets with rough sets has received intensive investigations [4,10,14-16,18-21,23,27,28,31-34,38,39,41,50,51]. However, most works focus on the fuzzy equivalence relations. So far as we know, there is no published work applying rough sets to approximate the membership functions of fuzzy sets.

Section 2 of this paper presents a brief overview of interval-valued fuzzy sets, Atanassov intuitionitic fuzzy sets, shadowed sets, type-2 fuzzy sets, rough sets and grey sets, defining the ideas and concepts that will be required for the rest of the paper. This section introduces the ideas informally to establish 
the semantics and then makes more formal definitions. Establishment of the semantics allows us to see what the definitions really mean, and also to grasp the differences. Section 3 defines R-fuzzy sets and gives some examples whilst Section 4 investigates the relationships between R-fuzzy sets and other fuzzy sets. Finally, Section 5 discusses the results.

\subsection{Motivation}

We use the voting model to generate membership functions across a set of populations. By performing various operations on this set of votes we obtain different fuzzy representations. At this point we are using just one criterion.

- The mean, mode or median of those membership functions give various ways of obtaining a conventional fuzzy set.

- The lower and upper bounds of the membership functions give interval valued fuzzy sets.

- If the question "what values would you not permit as a member" is also asked and used to derive a non-membership function we obtain Atanassov Intuitionistic Fuzzy Sets if we use the mean, mode or median; and by taking the interval given by the lower and upper bound of the voting sets we obtain Interval Valued Atanassov Intuitionistic Fuzzy Sets.

- The distribution of membership functions may be used to derive a fuzzy set to give type-2 fuzzy sets.

- Taking the higher values of membership functions as 1 and lower values as 0 , and leaving anything in between as $[0,1]$, we get shadowed sets.

- By taking those membership function values shared by all the voters as the lower approximation, and those values voted by at least one voter as the 
upper approximation, we get R-Fuzzy sets; the topic of this paper.

If we generate membership functions over a set of criteria we obtain L-fuzzy sets. The usual operations of conjunction and disjunction, along with others, may be the derived from the basic single criteria operations. So a conventional L-fuzzy set would have a vector of real numbers as its membership function. This membership function would be a precise vector with the representational accuracy available. Similarly, an interval valued L-fuzzy set would have a vector of intervals. It is important for the following that we understand that the vector has a fixed number of components in its representation of any given set. The accuracy of this vector in representing any criterion is governed by the accuracy of the representation used to measure the individual criteria.

By the nature of fuzziness, it is not always possible for people to give an accurate value for the membership value. However, it is feasible to give a set of permitted values in most cases. For example, if the membership of specific person in the set "tall" is to be evaluated , 30-40 voters may be in favour of $\{0.5,0.6,0.7\}$ and some other voters may take $\{0.6,0.7,0.8\}$ as their choice of the values. Here, every voter is in favour of 0.6 and 0.7 . Then we can represent the membership value of this person to be "tall" as $(\{0.6,0.7\},\{0.5,0.6,0.7$, $0.8\}$ ). Different from type-2, we do not need a precise secondary membership here, so there is no need to go for type- 3 which is needed to resolve the remaining imprecision of type-2. Compared with interval-valued fuzzy sets, we are not saying that 0.65 has to be a valid or candidate membership value unless we assume continuity. Hence we have a different form of information to interval-valued fuzzy sets. In this example we see that the inner approximation and the outer approximation may be represented as intervals. We may obtain a rough set membership function as $(\{0.5,0.8\},\{0.5,0.6,0.7,0.8\})$ where the 
lower approximation is disjoint and the central values would definitely not be considered as candidates for the lower approximation.

\section{Basic Definitions}

We first need to more formally define some relevant concepts in order to place the work into context and also to provide the necessary material to build the later work on.

Definition 1 (Fuzzy sets[52]) Let $\mathbb{U}$ denote a universe of discourse. Then a fuzzy set $A$ in $\mathbb{U}$ is defined as a set of ordered pairs

$$
A=\left\{\left\langle x, \mu_{A}(x)\right\rangle \mid x \in \mathbb{U}\right\}
$$

where $\mu_{A}: \mathbb{U} \longrightarrow[0,1]$ is the membership function of $A$ and $\mu_{A}(x)$ is the grade of belongingness of $x$ in $A$.

The membership function value [22] can be any real number between 0 and 1 which implies the vagueness caused by lack of sharp class boundaries. However, it is not always possible to give a crisp value for the membership. For instance, if the information about some membership values is not complete, a crisp value will be impossible to determine. Under such situations, the membership representation in definition 1 has to be modified to consider the secondary uncertainty involved. There have been several extensions of fuzzy sets to accommodate the uncertainties in fuzzy membership values. If the uncertain membership values of a fuzzy set could be represented as intervals between 0 and 1 , then we get the so called interval-valued fuzzy sets.

Definition 2 (Interval-valued fuzzy set[35]) Let $D[0,1]$ be the set of all 
closed subintervals of the interval $[0,1] . \mathbb{U}$ is the universe of discourse, $x$ is an element and $x \in X$. An interval-valued fuzzy set in $\mathbb{U}$ is given by set $A$

$$
A=\left\{\left\langle x, M_{A}(x)\right\rangle \mid x \in \mathbb{U}\right\}
$$

with $M_{A}: \mathbb{U} \rightarrow D[0,1]$.

The membership of an individual element is thus characterised as an interval instead of a single value in fuzzy sets. Interval-valued fuzzy sets provide a simple and efficient representation of the uncertain membership values of a fuzzy set [8]. Similar to interval-valued fuzzy sets, Atanassov intuitionistic fuzzy sets [1] provide another model for representing uncertainties in membership values of a fuzzy set.

Definition 3 (Atanassov intuitionistic fuzzy sets[1]) An Atanassov intuitionistic fuzzy set $A$ in $\mathbb{U}$ is given by

$$
A=\left\{\left\langle x, \mu_{A}(x), \nu_{A}(x)\right\rangle \mid x \in \mathbb{U}\right\}
$$

where

$$
\mu_{A}: \mathbb{U} \rightarrow[0,1], \quad \nu_{A}: \mathbb{U} \rightarrow[0,1]
$$

and

$$
0 \leq \mu_{A}(x)+\nu_{A}(x) \leq 1 \quad \forall x \in \mathbb{U}
$$

For each $x$, the numbers $\mu_{A}(x)$ and $\nu_{A}(x)$ are the degree of membership and degree of non-membership of $x$ in A respectively.

An Atanassov intuitionistic fuzzy set becomes a fuzzy set when $\nu_{A}(x)=1-$ $\mu_{A}(x)$. It can also be converted to an equivalent interval-valued fuzzy set. However, there are some semantic differences [7] between these two models. 
If the membership function of a fuzzy set involves uncertainties, it is reasonable to represent the membership function using a fuzzy set. We have a special fuzzy set whose membership function is described by fuzzy sets as well. Such a fuzzy set is called type-2 fuzzy set [25]. It is a general extension to fuzzy sets, and interval-valued fuzzy sets can be considered as a special case of type-2 fuzzy sets $[8]$.

Definition 4 (type-2 fuzzy sets[25]) A type-2 fuzzy set A, is characterized by a type-2 membership function $\mu_{A}(x, u)$, where $x \in \mathbb{U}$ and $u \in J_{x} \subseteq[0,1]$, $J_{x}$ is a finite discretised set which limits the values of $u \subseteq[0,1]$, i.e.,

$$
A=\left\{\left\langle(x, u), \mu_{A}(x, u)\right\rangle \mid \forall x \in \mathbb{U}, \forall u \in J_{x} \subseteq[0,1]\right\}
$$

in which $\mu_{A}: \mathbb{U} \times J_{x} \longrightarrow[0,1]$. A can also be expressed as

$$
A=\int_{x \in \mathbb{U}} \int_{u \in J_{x}} \mu_{A}(x, u) /(x, u) \quad J_{x} \subseteq[0,1]
$$

where $\iint$ denotes union over all admissible $x$ and $u$. For discrete universes of discourse $\int$ is replaced by $\sum$.

Rough sets take a different route from fuzzy sets in representing uncertainties. It represents an uncertain set by means of approximations in information systems. An information system is described by a pair $(\mathbb{U}, A)$, where $\mathbb{U}$ is a non-empty, finite set of objects called the universe and $A$ is a non-empty, finite set of attributes. Every attribute $a \in A$ of an object has a value. An attribute's value must be a member of the set $V_{a}$ which is called the value set of attribute $a\left(a: \mathbb{U} \rightarrow V_{a}\right)$.

Definition 5 (Approximation[30]) $\Lambda=(\mathbb{U}, A)$ is a given information system, $X \subseteq \mathbb{U}$ is a set. For a given set $B \subseteq A$, the set $X$ is approximated with 
two sets $B_{*}(X)$ and $B^{*}(X)$

$$
\begin{gathered}
B_{*}(X)=\bigcup_{x \in \mathbb{U}}\{B(x): B(x) \subseteq X\} \\
B^{*}(X)=\bigcup_{x \in \mathbb{U}}\{B(x): B(x) \cap X \neq \emptyset\}
\end{gathered}
$$

here, $B(x)$ refers to an equivalence class containing $x . B_{*}(X)$ and $B^{*}(X)$ are called the B-lower and B-upper approximations of $X$, respectively.

The $B$-lower approximation contains objects that are known to be members of $\mathrm{X}$. The objects in the set of the $B$-upper approximation are possible members of $\mathrm{X}$. In this paper, we adopt the set-oriented interpretation of rough sets $[19,26,29,47,50]$ and define a rough set as a pair of definable sets [19].

Definition 6 (Rough sets[50]) Let the pair apr $=(\mathbb{U}, B)$ be an approximation space on $\mathbb{U}$ and $\mathbb{U} / B$ denotes the set of all equivalence classes of $B$. The family of all definable sets in approximation space apr is denoted by Def(apr). Given two subsets $\underline{A}, \bar{A} \in \operatorname{Def}($ apr $)$ with $\underline{A} \subseteq \bar{A}$, the pair $(\underline{A}, \bar{A})$ is called a rough set.

Here, $\underline{A}$ is the lower approximation of $X=(\underline{A}, \bar{A})$, and $\bar{A}$ is the upper approximation of $X$. The boundary region could be derived as $\bar{A}-\underline{A}$. If $\bar{A}=\underline{A}$, then $(\underline{A}, \bar{A})$ is a definable set. For an element $x \in \mathbb{U}$, we have:

- if $x \in \underline{A}$ then $x \in(\underline{A}, \bar{A})$

- if $x \in \mathbb{U}-\bar{A}$ then $x \notin A$

- if $x \in \bar{A}$ and $x \notin \underline{A}$ then $\mathrm{x}$ has unknown relation with $(\underline{A}, \bar{A})$

It should be noted that different views exist for rough sets interpretation, so do their properties. A comprehensive review was given by Yao in [50]. Here, we adopt the Iwinsky type set-oriented rough sets [19], although there are other 
similar definitions of set-oriented rough sets, such as the P-rough sets defined by Pawlak [29,50] and the disjoint pair [26].

Rough sets and fuzzy sets are two different theories capturing two distinct aspects of imperfection in knowledge: indiscernibility and vagueness[14]. However, as a concept induced from fuzzy sets, shadowed sets [32] have a close relationship with rough sets as well. Considering a fuzzy set $A \in \mathbb{U}$, we elevate those membership values that are high enough to 1 and reduce those substantially low membership values to 0 , and represent those values in between as $[0,1]$, then we have transformed the fuzzy set to a shadowed set [32].

$$
A: \mathbb{U} \longrightarrow\{0,1,[0,1]\}
$$

Here, each element $x$ is associated with 0,1 or $[0,1]$. The elements for which $A(x)$ attains 1 constitute its core, and the elements where $A(x)=[0,1]$ form a shadow where uncertainty exists. Shadowed sets do not require precise membership values, and partition the elements of a fuzzy set into three categories: Yes (1), No (0) and Unknown $([0,1])$. In this sense, shadowed sets are conceptually close to rough sets.

As a different model for uncertainty representation, grey systems [9] provide another route to uncertainty modelling. In grey systems, the information is classified into three categories: white with completely certain information, grey with insufficient information, and black with totally unknown information. A grey number is a number with clear upper and lower boundaries but which has an unknown position within the boundaries[24]. Combining fuzzy sets and grey systems, we proposed grey sets as a general model for uncertainty 
representation[46,48,49].

Definition 7 (Grey sets[49]) For a set $A \subseteq \mathbb{U}$, if the characteristic function value of $x$ with respect to $A$ can be expressed with a continuous grey number $v^{ \pm}=\left[v^{-}, v^{+}\right] \in D[0,1]^{ \pm}$or a discrete grey number $v^{ \pm}=\left\{v^{-}, v_{1}, v_{2}, \ldots, v_{k}, v^{+}\right\} \in$ $D[0,1]^{ \pm}$

$$
\chi_{G}: \mathbb{U} \rightarrow D[0,1]^{ \pm}
$$

then $A$ is a grey set.

Here, $D[0,1]^{ \pm}$refers to the set of all grey numbers within the interval $[0,1]$. A grey number is different from an interval or a set in that it is a single number represented by an interval or a set [42]. Some recent research has linked grey sets with rough sets and proposed grey rough sets [40] for interval data. Although grey sets provide an alternative model for uncertainty representation, they have a close relationship with fuzzy sets $[46,48,49]$ and can be considered as an extension of fuzzy sets.

The dilemma of excessive precision in describing imprecise phenomena [32] has been resolved to some extent by interval-valued fuzzy sets, Atanassov intuitionistic fuzzy sets, shadowed sets, grey sets and type-2 fuzzy sets, but some outstanding questions remain. Interval-valued fuzzy sets, Atanassov intuitionistic fuzzy sets, shadowed sets and grey sets provide a convenient representation of the uncertainty involved in the membership values of a fuzzy set. However, the values within an interval may not be equally distributed as implied by interval-valued fuzzy sets. Some values may have a definite relationship with the membership values concerned, and some others may involve uncertainties. For example, some weather conditions are cold for everyone in- 
volved, but some other weather conditions may be considered as cold only by some individuals. Therefore, different people may give different temperatures as their understanding of "cold". There are both similarities and differences in their perceptions. It means that the fuzzy membership values for a descriptive object may not be representable by a single value or a single interval. An informative representation of a descriptive term should satisfy not only the requirements of imprecise representation, but also convey both the common perceptions and individual perceptions. Type-2 fuzzy sets have gone one step further to replace the crisp membership values of a fuzzy set with type-1 fuzzy sets. However, the secondary membership function of a type-2 fuzzy set is still described using crisp values, so we go back to the same dilemma. This dilemma exists even if we go to type-n fuzzy sets. However, as we regress downwards in our pursuit of precision we do get closer to an agreed model but at the expense of very heavy computation and complexity of representation. To solve this dilemma, it is necessary to describe the fuzzy membership function of a fuzzy set using a different model than fuzzy sets.

Fuzzy sets and rough sets are two different models of uncertainty representation [14]. Fuzzy sets focus on the ill-definition of the boundary of a concept by means of set characteristic functions. Rough sets highlight the indiscernibility between objects through equivalence relations. There have been many studies in the combination of these two models, such as fuzzy rough sets[14,23,27,51]. However, fuzzy rough sets adopt fuzzy sets to describe rough sets, and their representation is based on two fuzzy sets. A similar situation is true with rough fuzzy sets [51] as well. Most existing combination models define their sets by two fuzzy sets in the end. Here, we combine these two models in a different 
way, we describe the membership function of a fuzzy set using rough sets.

\section{$3 \quad$ R-fuzzy sets}

In general, a rough set is defined in a finite universe. It is necessary to discretise a continuous interval in $[0,1]$ into a discrete set. For a discrete set of values in $[0,1]$, we define a granule to measure its discretisation.

Definition 8 (Granule of a subset of values) Let $J_{x}=\left\{v_{1}, v_{2}, \ldots, v_{n}\right\}$ be a subset of values in $[0,1]$ and $v_{i} \leq v_{j}$ if $i \leq j$. The greatest difference $\Delta_{J_{x}}$ between two adjacent elements $v_{i}$ and $v_{i+1}(i<n)$ is called the granule of $J_{x}$ :

$$
\Delta_{J_{x}}=\max \left\{\left|v_{2}-v_{1}\right|,\left|v_{3}-v_{2}\right|, \ldots,\left|v_{n}-v_{n-1}\right|\right\}
$$

Obviously, if $J_{x}=\left[v_{1}, v_{1}\right]$, then we have $\Delta_{J_{x}}=0$.

Definition 9 Let $A=\left\{a_{1}, a_{2}, \ldots, a_{n}\right\}$ and $B=\left\{b_{1}, b_{2}, \ldots, b_{k}\right\}$ be two subsets of ordered values, where $a_{1} \leq a_{2} \leq \ldots \leq a_{n}$ and $b_{1} \leq b_{2} \leq \ldots \leq b_{k}$. We define

- $A \leq B$ iff $a_{1} \leq b_{1}$ and $a_{n} \leq b_{k}$

- $A<B$ iff $A \leq B$ but $A \neq B$

- $A \approx B$ iff $A \leq B$ and $B \leq A$

It should be noted here that $A \approx B$ is different from $A=B . A \approx B$ restricts only the bounds of the two sets, but they may contain other elements which are different between the two sets. 
Let pair apr $=\left(J_{x}, B\right)$ be an approximation space on a set of values $J_{x}=$ $\left\{v_{1}, v_{2}, \ldots, v_{n}\right\}$ and $J_{x} / B$ denotes the set of all equivalence classes of $B$. The family of all definable sets in approximation space apr is denoted by $\operatorname{Def}($ apr $)$. Given two subsets $\underline{M}, \bar{M} \in \operatorname{Def}(\operatorname{apr})$ with $\underline{M} \subseteq \bar{M}$, the pair $(\underline{M}, \bar{M})$ is obviously a rough set according to Definition 6 . Therefore, a subset of values could be approximated with a rough set when its exact boundary is not possible to be precisely identified using $J_{x} / \mathrm{B}$.

Considering $J_{x}$ as the universe of discrete values of possible fuzzy membership after discretisation of $[0,1]$, we have $J_{x} \subseteq[0,1]$. Under this situation, the granule $\Delta_{J_{x}}$ of $J_{x}$ has significant influence on the approximation of a subset of values in $[0,1]$. For the approximated membership values, its rough approximation $(\underline{M}, \bar{M})$ could be different with a different discretisation. However, it converges to a pair of intervals when its granule $\Delta_{J_{x}}$ approaches 0 :

$$
\lim _{\Delta_{J_{x}} \longrightarrow 0}(\underline{M}, \bar{M})=\left(\left[x_{1}, x_{2}\right],\left[y_{1}, y_{2}\right]\right)
$$

where $x_{1}=\inf \underline{M}_{\Delta_{J_{x}} \rightarrow 0}, x_{2}=\sup \underline{M}_{\Delta_{J_{x}} \rightarrow 0}, y_{1}=\inf \bar{M}_{\Delta_{J_{x}} \rightarrow 0}$ and $y_{2}=$ $\sup \bar{M}_{\Delta_{J_{x}} \rightarrow 0}$.

Now, we can define R-fuzzy sets.

Definition 10 (R-fuzzy sets) Let the pair apr $=\left(J_{x}, B\right)$ be an approximation space on a set of values $J_{x}=\left\{v_{1}, v_{2}, \ldots, v_{n}\right\} \subseteq[0,1]$ and $J_{x} / B$ denotes the set of all equivalence classes of $B .\left(M_{A}(x), \overline{M_{A}}(x)\right)$ be a rough set in apr. A R-fuzzy set $A$, is characterized by a rough set as its membership function $\left(\underline{M_{A}}(x), \overline{M_{A}}(x)\right)$, where $x \in \mathbb{U}$, i.e.,

$$
A=\left\{\left\langle x,\left(\underline{M_{A}}(x), \overline{M_{A}}(x)\right)\right\rangle \mid \forall x \in \mathbb{U}, \underline{M_{A}}(x) \subseteq \overline{M_{A}}(x) \subseteq J_{x}\right\}
$$


A can also be expressed as

$$
A=\sum_{x \in \mathbb{U}}\left(\underline{M_{A}}(x), \overline{M_{A}}(x)\right) / x
$$

where $\sum$ denotes union over all admissible $x$.

Similar to type-2 fuzzy sets and interval-valued fuzzy sets, a R-fuzzy set describes its membership using a set itself. Membership of R-fuzzy sets is a set of values satisfying its membership description. Therefore, the membership values of a R-fuzzy set usually contains more than one value although a single valued set is possible.

For each $x_{i} \in \mathbb{U}$, there is an associated membership description $d\left(x_{i}\right)$ describing the belongingness of $x_{i}$ to a set $A \subseteq \mathbb{U} . J_{x}$ is a given subset of values with possible membership values of a R-fuzzy set $A \subseteq \mathbb{U}$. $C$ is a set of available evaluation criteria. Each value $v \in J_{x}$ is evaluated by $c_{j} \in C$ to determine if it fits with $d\left(x_{i}\right)$ for $x_{i}$ with respect to $A$. The result of the evaluation is Yes or No, and we can represent this evaluation as:

$$
v \stackrel{\left(d\left(x_{i}\right), c_{j}\right)}{\longrightarrow} \text { Yes or } \quad v \stackrel{\left(d\left(x_{i}\right), c_{j}\right)}{\longrightarrow} N_{O}
$$

For each pair $\left(d\left(x_{i}\right), c_{j}\right)$ where $x_{i} \in \mathbb{U}$ and $c_{j} \in C$, a set $M_{c_{j}}\left(x_{i}\right) \subseteq J_{x}$ is constructed:

$$
M_{c_{j}}\left(x_{i}\right)=\left\{v \mid v \in J_{x}, v \stackrel{\left(d\left(x_{i}\right), c_{j}\right)}{\longrightarrow} Y e s\right\}
$$

The lower and upper approximations of the rough set $M\left(x_{i}\right)$ for the membership function described by $d\left(x_{i}\right)$ are

$$
\begin{aligned}
& \underline{M\left(x_{i}\right)}=\bigcap_{j} M_{c_{j}}\left(x_{i}\right) \\
& \overline{M\left(x_{i}\right)}=\bigcup_{j} M_{c_{j}}\left(x_{i}\right)
\end{aligned}
$$


Then the rough set approximating the uncertain membership $d\left(x_{i}\right)$ for $x_{i}$ is constructed as

$$
M\left(x_{i}\right)=\left(\bigcap_{j} M_{c_{j}}\left(x_{i}\right), \bigcup_{j} M_{c_{j}}\left(x_{i}\right)\right)
$$

For a given $d\left(x_{i}\right)$, it is obvious that $M\left(x_{i}\right)$ depends on $J_{x}$. For the same $d\left(x_{i}\right)$, $M_{a}\left(x_{i}\right)$ and $M_{b}\left(x_{i}\right)$ are two R-fuzzy memberships constructed for $A \subseteq \mathbb{U}$ from $J_{x}^{a}$ and $J_{x}^{b}$ using the same criteria set $C . M_{a}\left(x_{i}\right)$ and $M_{b}\left(x_{i}\right)$ may be different if $J_{x}^{a} \neq J_{x}^{b}$. For the same $C$, however, $M_{a}\left(x_{i}\right)=M_{b}\left(x_{i}\right)$ holds if $J_{x}^{a}=J_{x}^{b}$. Particularly, both $M_{a}\left(x_{i}\right)$ and $M_{b}\left(x_{i}\right)$ converge to the same representation when their granule $\Delta_{J_{x}}$ approaches 0 .

$$
\lim _{\Delta_{J_{x}^{a}} \rightarrow 0} M_{a}\left(x_{i}\right)=\lim _{\Delta_{J_{x}^{b}} \rightarrow 0} M_{b}\left(x_{i}\right)
$$

In addition to $J_{x}$, a change of the criteria set $C$ brings a different $M\left(x_{i}\right)$ as well. Considering $v \stackrel{\left(d\left(x_{i}\right), c_{j}\right)}{\longrightarrow} Y e s$, it is possible for a different $C$ to produce a different $M_{c_{j}}\left(x_{i}\right)$ and hence a different $M\left(x_{i}\right)$. Therefore, a R-fuzzy set $A \subseteq \mathbb{U}$ can only be constructed when $J_{x}$ and $C$ are known.

Different people may have different perceptions about the same object. The set of perceptions of people is one example of the criteria set $C$, and each person has their own criteria $c_{j} \in C$ in evaluating a given object. R-fuzzy sets provide a very convenient model to deal with such situations.

Example $f_{1}, f_{2}, f_{3}, f_{4}, f_{5}, f_{6}, f_{7}, f_{8}, f_{9}, f_{10}$ are 10 flights whose noise levels are recorded at an airport. Their corresponding noise levels are 10, 20, 30, 50, 40, $70,20,80,30$ and $60(\mathrm{dBA}) \cdot p_{1}, p_{2}, p_{3}, p_{4}, p_{5}$ and $p_{6}$ are 6 people at the same location. They gave their evaluations on the disturbances of these flights as shown in Table 1 . Here, VN, BN, AC and NN refer to very noisy (VN), a bit noisy (BN), acceptable (AC) and not noisy (NN). 
Table 1

Human perception of noise

\begin{tabular}{|c|c|c|c|c|c|c|c|c|c|c|}
\hline \hline person & $f_{1}$ & $f_{2}$ & $f_{3}$ & $f_{4}$ & $f_{5}$ & $f_{6}$ & $f_{7}$ & $f_{8}$ & $f_{9}$ & $f_{10}$ \\
\hline \hline$p_{1}$ & $\mathrm{NN}$ & $\mathrm{NN}$ & $\mathrm{NN}$ & $\mathrm{AC}$ & $\mathrm{AC}$ & $\mathrm{BN}$ & $\mathrm{NN}$ & $\mathrm{VN}$ & $\mathrm{NN}$ & $\mathrm{BN}$ \\
$p_{2}$ & $\mathrm{NN}$ & $\mathrm{NN}$ & $\mathrm{AC}$ & $\mathrm{AC}$ & $\mathrm{AC}$ & $\mathrm{BN}$ & $\mathrm{NN}$ & $\mathrm{VN}$ & $\mathrm{AC}$ & $\mathrm{BN}$ \\
$p_{3}$ & $\mathrm{NN}$ & $\mathrm{AC}$ & $\mathrm{AC}$ & $\mathrm{BN}$ & $\mathrm{AC}$ & $\mathrm{VN}$ & $\mathrm{AC}$ & $\mathrm{VN}$ & $\mathrm{AC}$ & $\mathrm{BN}$ \\
$p_{4}$ & $\mathrm{NN}$ & $\mathrm{NN}$ & $\mathrm{NN}$ & $\mathrm{AC}$ & $\mathrm{AC}$ & $\mathrm{BN}$ & $\mathrm{NN}$ & $\mathrm{VN}$ & $\mathrm{NN}$ & $\mathrm{BN}$ \\
$p_{5}$ & $\mathrm{NN}$ & $\mathrm{AC}$ & $\mathrm{AC}$ & $\mathrm{AC}$ & $\mathrm{AC}$ & $\mathrm{BN}$ & $\mathrm{AC}$ & $\mathrm{VN}$ & $\mathrm{AC}$ & $\mathrm{BN}$ \\
$p_{6}$ & $\mathrm{NN}$ & $\mathrm{NN}$ & $\mathrm{AC}$ & $\mathrm{AC}$ & $\mathrm{AC}$ & $\mathrm{VN}$ & $\mathrm{NN}$ & $\mathrm{VN}$ & $\mathrm{AC}$ & $\mathrm{BN}$ \\
\hline \hline
\end{tabular}

To construct a fuzzy set, we use noise level to establish its membership function. We apply the following simple linear function:

$$
\mu\left(f_{i}\right)=\frac{l_{i}-l_{\min }}{l_{\max }-l_{\min }}
$$

Here, $l_{i}$ refers to the noise level of $f_{i}, l_{\max }$ and $l_{\min }$ represent the maximum and minimum value of $l_{i}$. Therefore, we get the precise fuzzy membership values for the 10 flights:

$$
\begin{aligned}
& \mu\left(f_{1}\right)=0, \quad \mu\left(f_{2}\right)=0.14, \quad \mu\left(f_{3}\right)=0.29, \quad \mu\left(f_{4}\right)=0.57, \quad \mu\left(f_{5}\right)=0.43 \\
& \mu\left(f_{6}\right)=0.86, \quad \mu\left(f_{7}\right)=0.14, \quad \mu\left(f_{8}\right)=1, \quad \mu\left(f_{9}\right)=0.29, \quad \mu\left(f_{10}\right)=0.71
\end{aligned}
$$

However, it is not always possible to know the exact noise level of flights, and furthermore, people do not need to know the exact noise level in their communication. It raises a question to fuzzy sets: how to express the fuzzy 
membership function if we do not know the exact noise level? For example, if we know a flight $f_{11}$ whose noise is acceptable, how to describe it using fuzzy memberships? The traditional fuzzy sets will simply assign a precise membership value to it, such as 0.57 by $P_{1}$. However, Table 1 reveals that each person may give a different answer to this question. For example, $P_{5}$ may get 0.29 instead. It is the difference in the perception of people that makes their answers different. The difference in perception may cause misunderstanding in communication of our fuzzy concepts, so it is important to conserve it in our fuzzy membership. We can do this using R-fuzzy sets. In this example, $J_{x}$ is the set of all membership values in the 10 recorded flights. Therefore, we have

$$
J_{x}=\{0,0.14,0.29,0.43,0.57,0.71,0.86,1\}
$$

We know that $f_{11}$ is an "acceptable" flight, hence $d\left(f_{11}\right)=$ "acceptable" and its membership has to satisfy this description. Here, the evaluation criteria is decided by each person, $C=\left\{p_{1}, p_{2}, p_{3}, p_{4}, p_{5}, p_{6}\right\}$. Each value $v \in J_{x}$ is evaluated by $p_{j} \in C$ to determine if it fits with $d\left(f_{11}\right)$ for $f_{11} \in \mathbb{U}$.

$$
v \stackrel{\left(d\left(f_{11}\right), p_{j}\right)}{\longrightarrow} \text { Yes }
$$

For each $p_{i} \in C$, there is a corresponding row in Table 1. For those columns with a matching description with $d\left(f_{11}\right)=$ "AC", its corresponding flights will give the membership values according to their noise levels. Then we can construct a subset of values $M_{p_{j}}\left(f_{11}\right) \subseteq J_{x}$ according to Table 1 .

$$
\begin{array}{rrrr}
M_{p_{1}}\left(f_{11}\right)= & \{0.43,0.57\}, & M_{p_{2}}\left(f_{11}\right)= & \{0.29,0.43,0.57\} \\
M_{p_{3}}\left(f_{11}\right)= & \{0.14,0.29,0.43,0.57\}, & M_{p_{4}}\left(f_{11}\right)= & \{0.43,0.57\} \\
M_{p_{5}}\left(f_{11}\right)= & \{0.14,0.29,0.43,0.57\}, & M_{p_{6}}\left(f_{11}\right)= & \{0.29,0.43,0.57\}
\end{array}
$$

The lower and upper approximations of the rough set $M\left(f_{11}\right)$ for the mem- 
bership function described by $d\left(f_{11}\right)$ are

$$
\underline{M}\left(f_{11}\right)=\bigcap_{j} M_{p_{j}}\left(f_{11}\right) \text { and } \bar{M}\left(f_{11}\right)=\bigcup_{j} M_{p_{j}}\left(f_{11}\right)
$$

Then the rough set approximating the uncertain membership $d\left(f_{11}\right)$ is constructed as

$$
M\left(f_{11}\right)=\left(\bigcap_{j} M_{p_{j}}\left(f_{11}\right), \bigcup_{j} M_{p_{j}}\left(f_{11}\right)\right)
$$

Therefore, we have

$$
M\left(f_{11}\right)=(\{0.43,0.57\},\{0.14,0.29,0.43,0.57\})
$$

The results suggest that the membership values 0.43 and 0.57 are agreed by all people that their corresponding flights are "Acceptable". In addition to this, the flights associated with values 0.14 and 0.29 are also considered as "Acceptable" by some people although others may consider it as "Not Noisy" rather than "Acceptable".

To define the membership value of $f_{11}$, traditional type- 1 fuzzy sets will take a value like average value to represent it:

$$
\begin{aligned}
\mu\left(f_{11}\right)= & \frac{1}{23} \sum(0.57+0.43+0.29+0.57+0.43+0.29+0.14 \\
& +0.29+0.43+0.14+0.29+0.57+0.43+0.14+0.29+0.57 \\
& +0.43+0.14+0.29+0.29+0.57+0.43+0.29) \\
= & 0.36
\end{aligned}
$$

Obviously, this result suggests that 0.57 is not appropriate to describe "Acceptable", however, the data in Table 1 shows that every one agrees on the validity of 0.57 as a description for "Acceptable". Another possible solution here is to apply interval-valued fuzzy sets. Considering the lower and upper 
bounds of the membership values for "Acceptable" in Table 1, "Acceptable" can be represented by $[0.14,0.57]$. It does include all possibilities now, but one cannot tell which values are shared by all people. This important information is lost as a result of interval-valued fuzzy sets. Intuitionistic fuzzy sets share the same problems. For type-2 fuzzy sets, the limited number of flights here is not enough to setup a reliable membership distribution, and furthermore, the computational complexity is much higher than other models. Shadowed sets would consider "Acceptable" as unknown, so it cannot help this situation. Obviously, R-fuzzy sets provide a unique tool here.

For rough set $M\left(f_{11}\right)$, the lower approximation of membership values represents those values agreed by each person. If a value belongs to the lower approximation, then the value definitely satisfies its membership description. However, $M\left(f_{11}\right)$ does not exclude the existence of other possible perceptions not shared by other people, and it represents them by means of the upper approximation of membership values. A value in the upper approximation indicates that it is possible for the value to belong to the described membership depending on different individuals. Therefore, $M\left(f_{11}\right)$ recognises and conserves the diversity of perceptions in its fuzzy membership representations. This kind of information may be important for airports and the residences in their vicinity. A model ignoring the diversity of perceptions may give false noise exposure annoyance than it is in the real world [17]. As aforementioned, a model using type-2 fuzzy sets here loses information on the applicability of flights associated with 0.57 , and a model with interval-valued fuzzy sets does not differentiate the applicability between 0.57 and 0.14 . In real world airport operations, a target of 0.57 is much more applicable than 0.36 or 0.14 . A rep- 
resentation using R-fuzzy sets is especially beneficial when different airports are involved. The aircraft noise perceptions could be significantly different between two airports even if their geographical locations are not far away from each other, and a representation using R-fuzzy sets could naturally reveal the difference. Obviously, this application could be extended into other areas such as communications between different communities with different geographical locations or subjects.

A R-fuzzy set describes its membership function with a rough set. The involvement of rough approximations in membership functions provides the capability for R-fuzzy sets to represent some situations, which are difficult to model by other fuzzy sets. Other techniques have been used to model aircraft noise as it is highly relevant to modern society and especially to those living and working near airports [43-45].

Example $\mathbb{U}=\left\{x_{1}, x_{2}, \ldots, x_{n}\right\}$ is a set of voters for party $\mathrm{A}$ and party $\mathrm{B}$. The political preference of voters can be represented as a set: \{support A, close to A, slightly bias to A than B, no preference, slightly bias to B than A, close to B, support B\}. We can convert this preference set into a set of values

$$
J_{x}=\{1,0.84,0.67,0.5,0.34,0.17,0\}
$$

If we know the exact preference of each voter, then we can represent their preference using a fuzzy set

$$
S=\sum_{x \in \mathbb{U}} v / x \quad v \in J_{x}
$$

Sometimes, we may not know the exact preference for some voters. For example, we know that $x_{2}$ has a preference to a party although they are not a 
supporter of any party. Under this situation, $x_{2}$ may prefer A or B but we have no certain information on this, but we do know that they are neither a supporter of a party nor a voter without preference. Considering the existing fuzzy set models, such as type-1 fuzzy sets, type-2 fuzzy sets, interval-valued fuzzy sets, Atanassov intuitionistic fuzzy sets and shadowed sets, none of these could represent this situation. However, the proposed R-fuzzy sets can easily represent it:

$$
S=\sum_{x \in \mathbb{U}}(\underline{M}(x), \bar{M}(x)) / x
$$

For the aforementioned $x_{2} \in \mathbb{U}$, the uncertain membership description $d\left(x_{2}\right)=$ " $x_{2}$ prefers one party but does not support any party". Considering two possible preferences: $a$ for party $\mathrm{A}$ and $b$ for party $\mathrm{B}$, we have two elements in the criteria set $C=\{a, b\}$. Each value $v \in J_{x}$ is evaluated by $c_{j} \in C$ to determine if it fits with $d\left(x_{2}\right)$.

$$
v \stackrel{\left(d\left(x_{2}\right), c_{j}\right)}{\longrightarrow} Y e s
$$

For each element $c_{j} \in C$, a set $M_{c_{j}}\left(x_{2}\right) \subseteq J_{x}$ is constructed:

$$
M_{a}\left(x_{2}\right)=\{0.84,0.67\}, \quad M_{b}\left(x_{2}\right)=\{0.34,0.17\}
$$

The lower and upper approximations of rough set $M\left(x_{2}\right)$ for the membership function described by $d\left(x_{2}\right)$ are

$$
\underline{M}\left(x_{2}\right)=\bigcap_{j} M_{c_{j}}\left(x_{2}\right) \quad \text { and } \quad \bar{M}\left(x_{2}\right)=\bigcup_{j} M_{c_{j}}\left(x_{2}\right)
$$

Then we have

$$
M\left(x_{2}\right)=(\emptyset,\{0.84,0.67,0.34,0.17\})
$$

Considering a different criteria set $C=\{h, s\}$ where $h$ refers to a high preference and $s$ represents a preference which is at least weak, for the same 
description $d\left(x_{2}\right)$ we have

$$
M_{h}\left(x_{2}\right)=\{0.84,0.17\}, \quad M_{s}\left(x_{2}\right)=\{0.84,0.67,0.34,0.17\}
$$

Then

$$
M\left(x_{2}\right)=(\{0.84,0.17\},\{0.84,0.67,0.34,0.17\})
$$

Clearly, the criteria set has a significant influence on the approximation of the membership of a R-fuzzy set.

Both examples demonstrate that the proposed R-fuzzy sets can represent some situations that are difficult to be expressed using the existing extensions of fuzzy sets. R-fuzzy sets provide a new alternative in expressing uncertain fuzzy memberships of a fuzzy set. Its difference from other fuzzy sets inevitably introduces different operations into R-fuzzy sets. The membership of an element in a R-fuzzy set is defined as a rough set, hence its operation result is defined by the pair of definable sets for the rough approximation of its membership.

Definition $11 A$ and $B$ are two $R$-fuzzy sets of a universe $\mathbb{U}, \forall x \in \mathbb{U}$

- $A \subseteq B$ iff $\underline{M}_{A}(x) \leq \underline{M}_{B}(x)$ and $\bar{M}_{A}(x) \leq \bar{M}_{B}(x)$

- $A=B$ iff $\underline{M}_{A}(x)=\underline{M}_{B}(x)$ and $\bar{M}_{A}(x)=\bar{M}_{B}(x)$

- $\bar{M}_{\neg A}(x)=J_{x}-\underline{M}_{A}(x)$ and $\underline{M}_{\neg A}(x)=J_{x}-\bar{M}_{A}(x)$

- $\underline{M}_{A \cup B}(x)=\left\{m \in \underline{M}_{A}(x) \cup \underline{M}_{B}(x) \mid m \geq \max \left\{\inf \underline{M}_{A}(x), \inf \underline{M}_{B}(x)\right\}\right\}$ and $\bar{M}_{A \cup B}(x)=\left\{m \in \bar{M}_{A}(x) \cup \bar{M}_{B}(x) \mid m \geq \max \left\{\inf \bar{M}_{A}(x), \inf \bar{M}_{B}(x)\right\}\right\}$

- $\underline{M}_{A \cap B}(x)=\left\{m \in \underline{M}_{A}(x) \cup \underline{M}_{B}(x) \mid m \leq \min \left\{\sup \underline{M}_{A}(x), \sup \underline{M}_{B}(x)\right\}\right\}$ and $\bar{M}_{A \cap B}(x)=\left\{m \in \bar{M}_{A}(x) \cup \bar{M}_{B}(x) \mid m \leq \min \left\{\sup \bar{M}_{A}(x), \sup \bar{M}_{B}(x)\right\}\right\}$

- $\underline{M}_{\perp A}(x)=\bar{M}_{\perp A}(x)$

- $\left(\underline{M}_{\oslash A}(x), \bar{M}_{\oslash A}(x)\right) \in\left\{(\{0\},\{0\}),(\{1\},\{1\}),\left(\emptyset, J_{x}\right)\right\}$

- $\underline{M}_{\infty A}(x)=\lim _{\Delta_{J_{x}} \rightarrow 0} \underline{M}_{A}(x)$ and $\bar{M}_{\infty A}(x)=\lim _{\Delta_{J_{x}} \rightarrow 0} \bar{M}_{A}(x)$ 
- $\left|\underline{M}_{\angle A}(x)\right|=1$ and $\underline{M}_{\angle A}(x) \leq \bar{M}_{\angle A}(x)$

- $\underline{M}_{\diamond A}(x)=\emptyset$ and $\bar{M}_{\diamond A}(x) \neq \emptyset$

Here, $\neg A$ is the complement set of $A$ in $\mathbb{U}, \perp A$ has no unknown membership values, $\oslash A$ represents a set satisfying three-valued logic [6], $\infty A$ is a set with continuous membership representation and $\angle A$ has one single known membership value and each unknown membership value is known to be greater than the known value. With these operation definitions, we can consider the relationship between R-fuzzy sets and other fuzzy sets.

R-fuzzy sets are different from ordinary fuzzy sets in that their membership values are expressed with a set rather than a value. Because of this set representation, its operation has to consider the involvement of the set as well. If $c_{1}$ and $c_{2}$ are two criteria, or information sources, then ordinary fuzzy sets can be represented as $c_{1}(A(x))$ and $c_{2}(A(x))$, and the corresponding $\mathrm{R}$-fuzzy sets are represented as $c_{1} c_{2}(A(x))$. In Definition 11, the definitions of $\cup$ and $\cap$ are the operations between two R-fuzzy sets rather than two ordinary fuzzy sets. Therefore, our definition is about $c_{1} c_{2}(A(x)) \cup c_{1} c_{2}(B(x))$. For example let us assume $c_{1}(A(x))=\{0.1\}, c_{2}(A(x))=\{0.3\}, c_{1}(B(x))=\{0.2\}$, and $c_{2}(B(x))=\{0.4\}$. Then accordingly, $\bar{M}_{A}(x)=\{0.1,0.3\}$ and $\bar{M}_{B}(x)=\{0.2,0.4\}$. It follows that $c_{1}((A \cup B)(x))=\{\max (0.1,0.2)\}=\{0.2\}$ and $c_{2}((A \cup B)(x))=\{0.4\}$ if $c_{1}$ and $c_{2}$ follow the rules of classical fuzzy set theory and we ignore the interactions in R-Fuzzy sets. Thus, $\bar{M}_{A \cup B}(x)=\{0.2,0.4\}$. However, according to Definition $11, \bar{M}_{A \cup B}(x)=\{0.2,0.3,0.4\}$. If $c_{1}$ and $c_{2}$ are used to evaluate $A$ and $B$, then there is no reason why $c_{1}(A(x)) \cup c_{2}(B(x))$ cannot be included in $c_{1} c_{2}(A(x)) \cup c_{1} c_{2}(B(x))$. If situations like this are included, we would have $\bar{M}_{c_{1} c_{2}(A) \cup c_{1} c_{2}(B)}(x)=\{0.2,0.3,0.4\}$. Obviously, $c_{1} c_{2}(A(x)) \cup c_{1} c_{2}(B(x))$ is different from $c_{1} c_{2}(A(x) \cup B(x))$. They are two different concepts and cannot 
be mixed. This example could also be examined intuitively by asking what could be the set of possible memberships given the sets of possible memberships in the constituent sets. Interpreting possible memberships for $\cup$, then from $c_{1}(B(x))=\{0.2\}$ gives a possible membership and $c_{2}(A(x))=\{0.3\}$ is another so $c_{1} c_{2}(A \cup B(x))=\{0.3\}$ is a possible membership. Hence R-Fuzzy sets capture this, whereas a reductionist approach misses this value.

\section{Relationship between R-fuzzy sets and other fuzzy sets}

A R-fuzzy set can represent some situations that are difficult to represent using other fuzzy sets, hence it is different from those. However, it overlaps with other fuzzy sets under some special situations.

Theorem $1 A R$-fuzzy set $A$ is a type-1 fuzzy set iff $A$ satisfies both $\angle A$ and $\perp A$.

Proof From definition 11, if $A$ satisfies both $\angle A$ and $\perp A$, we have $\left|\underline{M}_{A}(x)\right|=$ 1 and $\underline{M}_{A}(x)=\bar{M}_{A}(x)$. Hence, $\left|\underline{M}_{A}(x)\right|=\left|\bar{M}_{A}(x)\right|=1$, and we have

$$
A=\sum_{x \in \mathbb{U}}(\{\mu(x)\},\{\mu(x)\}) / x
$$

Both lower and upper approximations contain only a single element which is the same, so this is actually a crisp value, and is equivalent to a single value. Then we have

$$
A=\sum_{x \in \mathbb{U}} \mu(x) / x
$$

Obviously, it is a type-1 fuzzy set.

If $A$ is a type- 1 fuzzy set, we can replace its single membership value with a 
pair of identical single element sets

$$
A=\sum_{x \in \mathbb{U}}(\{\mu(x)\},\{\mu(x)\}) / x
$$

Thus, $\left|\underline{M}_{A}(x)\right|=1$ and $\underline{M}_{A}(x)=\bar{M}_{A}(x)$. From definition $11, A$ satisfies both $\angle A$ and $\perp A$.

Theorem $2 A R$-fuzzy set $A$ is an Atanassov intuitionistic fuzzy set iff $A$ satisfies both $\angle A$ and $\infty A$.

Proof If $A$ satisfies both $\angle A$ and $\infty A$, we have $\left|M_{A}(x)\right|=1$ and $\Delta_{J_{x}} \rightarrow 0$. Hence, $\bar{M}_{A}(x)=\lim _{\Delta_{J_{x}} \rightarrow 0} \bar{M}_{A}(x)=\left[\inf \bar{M}_{A}(x), \sup \bar{M}_{A}(x)\right]$, and we have

$$
A=\left\{\left\langle x,\left(\{\mu(x)\},\left[\inf \bar{M}_{A}(x), \sup \bar{M}_{A}(x)\right]\right)\right\rangle \mid \forall x \in \mathbb{U}\right\}
$$

For $\angle A$, we have $\underline{M}_{\angle A}(x) \leq \bar{M}_{\angle A}(x)$, thus

$$
A=\left\{\left\langle x,\left(\{\mu(x)\},\left[\mu(x), \sup \bar{M}_{A}(x)\right]\right)\right\rangle \mid \forall x \in \mathbb{U}\right\}
$$

The non-membership of set A could be derived from its membership:

$$
\left(1-\sup \bar{M}_{A}(x),\left[1-\sup \bar{M}_{A}(x), 1-\mu(x)\right]\right)
$$

The approximations of membership and non-membership overlap in their boundary area. If the lower approximations of membership and non-membership are fixed, then their upper approximations are fixed as well. Hence, we can represent the same set with the low approximations of membership and nonmembership.

$$
A=\left\{\left\langle x, \mu(x), 1-\sup \bar{M}_{A}(x)\right\rangle \mid \forall x \in \mathbb{U}\right\}
$$

Here, $\mu(x) \in[0,1], \mu(x) \leq \sup \bar{M}_{A}(x) \leq 1$, we have

$$
\mu(x)+\left(1-\sup \bar{M}_{A}(x)\right) \leq 1
$$


Obviously, $A$ is an Atanassov intuitionistic fuzzy set.

If $A$ is an Atanassov intuitionistic fuzzy set, we can replace its single nonmembership value with an approximation of the upper membership values

$$
A=\sum_{x \in \mathbb{U}}(\{\mu(x)\},[\mu(x), 1-\nu(x)]) / x
$$

Thus, $\left|\underline{M}_{A}(x)\right|=1$ and $\Delta_{J_{x}} \rightarrow 0$. From definition 11, $A$ satisfies both $\angle A$ and $\infty A$.

Theorem 3 A R-fuzzy set $A$ is an interval-valued fuzzy set iff $A$ satisfies both $\perp A$ and $\infty A$.

Proof For $\perp A$ and $\infty A$, we have $\underline{M}_{A}(x)=\bar{M}_{A}(x)=S(x)$ and $\Delta_{J_{x}} \rightarrow 0$. Hence, $S(x) \in D[0,1]$, and we have

$$
A=\{\langle x,(S(x), S(x))\rangle \mid \forall x \in \mathbb{U}\}
$$

Both lower and upper approximations contain exactly the same elements, so this is actually a crisp set. Then we have

$$
A=\{\langle x, S(x)\rangle \mid \forall x \in \mathbb{U}\}
$$

Obviously, it is an interval-valued fuzzy set.

If $A$ is an interval-valued fuzzy set, we can replace its crisp membership set (interval) with a pair of identical sets

$$
A=\{\langle x,(S(x), S(x))\rangle \mid \forall x \in \mathbb{U}\}
$$

Thus, $\underline{M}_{A}(x)=\bar{M}_{A}(x)=S(x)$ and $\Delta_{J_{x}} \rightarrow 0$. From definition 11, $A$ satisfies both $\perp A$ and $\infty A$. 
Interval-valued fuzzy sets $[35,36]$ and Atanassov intuitionistic fuzzy sets $[1-$ 3] have been proven to be mathematically equivalent [5,11-13,37]. However, they emerged from different grounds and thus they have associated different semantics [7]. Theorem 2 and 3 show their different interpretations in R-fuzzy sets. Interval-valued fuzzy sets can be considered as a special case of type- 2 fuzzy sets where the secondary grades are all equal to $1[8,25]$. It proves that all elements in the interval belong to the lower approximation of membership in R-fuzzy sets. For Atanassov intuitionistic fuzzy sets, its known membership is a single value, and the interval between the membership and possibility derived from the non-membership is considered as hesitation or uncertainty of the membership. Therefore, the interval represents the upper approximation in R-fuzzy sets, and the lower approximation of an Atanassov intuitionistic fuzzy set is represented as a set with a single element in R-fuzzy sets. When the lower approximation is empty, we get a grey set $[46,48,49]$.

Theorem 4 A R-fuzzy set $A$ is a grey set iff $A$ satisfies $\diamond A$ and $\left|\bar{M}_{A} \cap M_{A}\right|=$ 1.

Proof If $A$ satisfies $\diamond A$, we have $\underline{M}_{A}(x)=\emptyset$ and $\bar{M}_{A}(x) \neq \emptyset$. Considering $\left|\bar{M}_{A}(x) \cap M_{A}(x)\right|=1, M_{A}(x)$ represents a set with a single number $v_{w}(x)$. The single number $v_{w}(x)$ can only come from one element of $\bar{M}_{A}(x)$, we have

$$
\inf \bar{M}_{A}(x) \leq v_{w}(x) \leq \sup \bar{M}_{A}(x)
$$

Then, $\bar{M}_{A}(x) \in D[0,1]^{ \pm}$is a grey number. Hence, $A$ is a grey set.

If $A$ is a grey set, then $M_{A}(x)$ is a grey number. This grey number could be approximated by a rough set $\left(\underline{M}_{A}(x), \bar{M}_{A}(x)\right)$. The underlying white number is not known, we have $\underline{M}_{A}(x)=\emptyset$ and $\bar{M}_{A}(x) \neq \emptyset$. From definition 11 , this 
satisfies $\diamond A$. According to the nature of grey numbers, there is only a single white number underlying a grey number, hence we have $\left|\bar{M}_{A} \cap M_{A}\right|=1$.

Shadowed sets can be proved to be a special case of R-fuzzy sets as well.

Theorem 5 A R-fuzzy set $A$ is a shadowed set iff $A$ satisfies both $\oslash A$ and $\infty A$.

Proof If A satisfies $\oslash A$, we have

$$
\left(\underline{M}_{A}(x), \bar{M}_{A}(x)\right) \in\left\{(\{0\},\{0\}),(\{1\},\{1\}),\left(\emptyset, J_{x}\right)\right\}
$$

For $\infty A$, we have

$$
J_{x}=[0,1]
$$

Thus

$$
\left(\underline{M}_{A}(x), \bar{M}_{A}(x)\right) \in\{(\{0\},\{0\}),(\{1\},\{1\}),(\emptyset,[0,1])\}
$$

Obviously, for each $x \in \mathbb{U}$, there are only three situations for their fuzzy membership values:

$$
A: \mathbb{U} \longrightarrow\{0,1,[0,1]\}
$$

Clearly, this is a shadowed set.

If $\mathrm{A}$ is a shadowed set, we have

$$
A: \mathbb{U} \longrightarrow\{0,1,[0,1]\}
$$

Represent this mapping in rough sets

$$
A: \mathbb{U} \longrightarrow\{(\{0\},\{0\}),(\{1\},\{1\}),(\emptyset,[0,1])\}
$$

Therefore, we have

$$
\left(\underline{M}_{A}(x), \bar{M}_{A}(x)\right) \in\{(\{0\},\{0\}),(\{1\},\{1\}),(\emptyset,[0,1])\}
$$


Obviously, it satisfies both $\oslash A$ and $\infty A$.

It should be noted that we use $(\emptyset,[0,1])$ rather than $([0,1],[0,1])$ to represent unknown values. There is a difference between the two representations. $([0,1],[0,1])$ can be interpreted as we know for sure that each value in $[0,1]$ satisfies some description for the membership. It does not give the accurate meaning of the shadowed zone defined by Witold Pedrycz in [32]. With $(\emptyset,[0,1])$, it is clear that we know nothing about the value but its domain.

Interval-valued fuzzy sets could be considered as a special case of type-2 fuzzy sets, so theorem 3 gives some indication of the relationship between R-fuzzy sets and type-2 fuzzy sets under special situations. In fact, R-fuzzy sets and type-2 fuzzy sets are two different extensions to fuzzy sets, and they can not simply replace each other.

\section{Conclusions}

The fuzzy membership function of a fuzzy set has great significance in defining a fuzzy set. It is not possible to give a precise membership value sometimes. The uncertainties in fuzzy membership values have brought in various extensions of fuzzy sets, such as interval-valued fuzzy sets, Atanassov intuitionistic fuzzy sets, shadowed sets, type-2 fuzzy sets etc. Here, we defined R-fuzzy sets as a new extension of fuzzy sets, and derived its relationships with other fuzzy sets. Our results prove that R-fuzzy sets provide new facility in representing some situations which are difficult to describe in other models. The proposed R-fuzzy sets provide a new semantic context for the difference be- 
tween interval-valued fuzzy sets and Atanassov intuitionistic fuzzy sets also. Although they are mathematically equivalent in operation, R-fuzzy sets give a different perspective.

\section{References}

[1] K. Atanassov. Intuitionistic fuzzy sets. Physica-Verlag, Heidelberg - New York, 1999.

[2] K. T. Atanassov. Intuitionistic fuzzy sets. Fuzzy sets and systems, 20:87-96, 1986.

[3] K. T. Atanassov. More on intuitionistic fuzzy sets. Fuzzy sets and systems, $33: 37-46,1989$.

[4] S. Bodjanova. Granulation of a fuzzy set: Nonspecificity. Information Sciences, 177(20):4430-4444, 2007.

[5] H. Bustince and P. Burillo. Vague sets are intuitionistic fuzzy sets. Fuzzy Sets and Systems, 79:403-405, 1996.

[6] E. F. Codd. Missing information (applicable and inapplicable) in relational databases. SIGMOD Record, 15(4):53-78, 1986.

[7] C. Cornelis, K. T. Atanassov, and E. E. Kerre. Intuitionistic fuzzy sets and interval-valued fuzzy sets: a critical comparison. In M. Wagenknecht and R. Hampel, editors, Third EUSFLAT proceedings, pages 159-163, Zittau, Germany, September 2003. European Society for Fuzzy Logic and Technology.

[8] C. Cornelis, G. Deschrijver, and E. E. Kerre. Advances and challenges in interval-valued fuzzy logic. Fuzzy Sets and Systems, 157(5):622-627, 2006. 
[9] J. Deng. Control problems of grey systems. Systems and Control Letters, 1(5):288-294, 1982.

[10] T. Deng, Y. Chen, W. Xu, and Q. Dai. A novel approach to fuzzy rough sets based on a fuzzy covering. Information Sciences, 177(11):2308-2326, 2007.

[11] G. Deschrijver and E. Kerre. On the relationship between some extensions of fuzzy set theory. Fuzzy sets and systems, 133(2):227-235, 2003.

[12] D. Dubois, W. Ostasiewicz, and H. Prade. Fuzzy set: history and basic notions. In D. Dubois and H. Prade, editors, Fundamentals of fuzzy sets, pages 21-124. Kluwer, 2000.

[13] D. Dubois and H. Prade. Fuzzy sets and systems: theory and applications. Academic Press, New York, 1980.

[14] D. Dubois and H. Prade. Rough sets and fuzzy rough sets. International journal of general systems, 17:191-209, 1990.

[15] D. Dubois and H. Prade. Fuzzy relation equations and causal reasoning. Fuzzy sets and systems, 75:119-134, 1995.

[16] D. Dubois, H. Prade, F. Esteva, P. Garcia, and L. Godo. A logical approach to interpolation based on similarity relations. International Journal of Approximate Reasoning, 17:1-36, 1997.

[17] S. Fidell, L. Silvati, and E. Haloby. Social survey of community response to a step change in aircraft noise exposure. Journal of the Acoustical Society of America, 111:200-209, 2002.

[18] V.-N. Huynh and Y. Nakamori. A roughness measure for fuzzy sets. Information Science, 173(1):255-275, 2005.

[19] T. B. Iwinski. Algebraic approach to rough sets. Bull. Polish Acad. Sci. Math., 35:673-683, 1987. 
[20] R. Jensen and Q. Shen. Fuzzy rough attribute reduction with application to web categorization. Fuzzy sets and systems, 141:469-485, 2004.

[21] J. Jiang, C. Wu, and D. Chen. The product structure of fuzzy rough sets on a group and the rough t-fuzzy group. Information Sciences, 175(1):97-107, 2005.

[22] G. J. Klir and B. Yuan. Fuzzy sets and fuzzy logic theory and applications. Prentice Hall P T R, Upper Saddle River - New Jersey, 2002.

[23] J. Komorowski, J. Z. Pawlak, L. Polkowski, and A. Skowron. Rough sets: a tutorial. In S. K. Pal and A. Skowron, editors, Rough fuzzy hybridization: a new trend in decision-making, pages 3-98. Springer, Singapore, 1999.

[24] S. Liu, T. Gao, and Y. Dang. Grey systems theory and its applications. The Science Press of China, Beijing, 2000.

[25] J. Mendel and R. John. Type-2 fuzzy sets made simple. IEEE Transactions on Fuzzy Systems, 10(2):117-127, 2002.

[26] A. Mousavi and P. Jabedar-Maralani. Double-faced rough sets and rough communication. Information Science, 148:41-53, 2002.

[27] S. Nanda and S. Majumdar. Fuzzy rough sets. Fuzzy sets and systems, 45:157$160,1992$.

[28] A. Obtulowicz. Rough sets and heyting algebra valued sets. Bull. Polish Acad. Sci. Math., 35:667-671, 1987.

[29] Z. Pawlak. Rough sets. International journal of Computer and Information Sciences, 11(5):341-356, 1982.

[30] Z. Pawlak. Rough sets and intelligent data analysis. Information Science, $147: 1-12,2002$.

[31] Z. Pawlak and A. Skowron. Rough sets: Some extensions. Information Sciences, 177(1):28-40, 2007. 
[32] W. Pedrycz. Shadowed sets: representing and processing fuzzy sets. IEEE Transactions on Systems, Man, and Cybernetics - Part B: Cybernetics, 28:103109, 1998.

[33] A. Radzikowska and E. Kerre. A comparative study of fuzzy rough sets. Fuzzy sets and systems, 126:137-155, 2002.

[34] J. F. Salido and S. Murakami. Rough set analysis of a general type of fuzzy data using transitive aggregations of fuzzy similarity relations. Fuzzy sets and systems, 139:515-542, 2003.

[35] R. Sambuc. Fonctions $\Phi$-floues. Application I'Aide au Diagnostic en Patholologie Thyroidienne. PhD thesis, Univ. Marseille, France, 1975.

[36] B. Turksen. Interval valued fuzzy sets based on normal forms. Fuzzy Sets and Systems, 20:191-210, 1986.

[37] G. Wang and Y. He. Intuitionistic fuzzy sets and l-fuzzy sets. Fuzzy Sets and Systems, 110:271-274, 2000.

[38] A. Wiweger. On topological rough sets. Bull. Polish Acad. Sci. Math., 37:89-93, 1989.

[39] W. Wu, J. Mi, and W. Zhang. Generalized fuzzy rough sets. Fuzzy sets and systems, 151:263-282, 2003.

[40] D. Yamaguchia, G.-D. Lia, and M. Nagai. A grey-based rough approximation model for interval data processing. Information Sciences, 177(21):4727-4744, 2007.

[41] X. P. Yang. Minimization of axiom sets on fuzzy approximation operators. Information Sciences, 177(18):3840-3854, 2007.

[42] Y. Yang. Extended grey numbers and their operations. In Proceedings of 2007 IEEE International Conference on Ssystems, Man and Cybernetics, pages 
2181-2186, Montreal, Canada, October 2007.

[43] Y. Yang, D. Gillingwater, and C. Hinde. Applying neural networks and geographical information systems to airport noise evaluation. In Advances in Neural Networks: Proceedings of the Second International Symposium on Neural Networks, pages 998-1002. Springer-Verlag, 2001.

[44] Y. Yang, D. Gillingwater, and C. Hinde. An intelligent audit system for the sustainable development of airports. In 9th World Conference on Transport Research, pages 1-15, 2001.

[45] Y. Yang, C. Hinde, and D. Gillingwater. Airport noise simulation using neural networks. In 2008 IEEE World Congress on Computational Intelligence, IJCNN, pages 1918-11924. IEEE, 2008.

[46] Y. Yang and R. John. Grey systems and interval valued fuzzy sets. In M. Wagenknecht and R. Hampel, editors, Third EUSFLAT proceedings, pages 193-197, Zittau, Germany, September 2003. European Society for Fuzzy Logic and Technology.

[47] Y. Yang and R. John. Roughness bounds in rough set operations. Information Science, 176:3256-3267, 2006.

[48] Y. Yang, R. John, and F. Chiclana. Grey sets: a unified model for fuzzy sets and rough sets. In C. Hinde, editor, UKCI04 proceedings, pages 239-246, Loughborough, United Kingdom, September 2004.

[49] Y. Yang, R. John, and F. Chiclana. Grey sets, fuzzy sets and rough sets. In In Proceedings of the 5th International Conference on Recent Advances in Soft Computing, RASC 2004, Nottingham, UK, 16-18 December 2004, pages 348353, 2004. (ISBN 1-84233-110-8).

[50] Y. Y. Yao. Two views of the theory of rough sets in finite universe. International journal of approximate reasoning, 15(4):291-317, 1996. 
[51] Y. Y. Yao. Combination of rough and fuzzy sets based on alpha level sets. In T. Y. Lin and N. Cercone, editors, Rough sets and data mining: analysis for imprecise data, pages 301-321. Kluwer Academic, Boston, MA, 1997.

[52] L. Zadeh. Fuzzy sets. Information and Control, 8:338-353, 1965. 